\title{
Peroral Cholangioscopy-guided Electrohydraulic Lithotripsy with a SpyGlass DS Versus a Conventional Digital Cholangioscope for Difficult Bile Duct Stones
}

\author{
Toji Murabayashi, Takahisa Ogawa, Shinsuke Koshita, Yoshihide Kanno, Hiroaki Kusunose, \\ Toshitaka Sakai, Kaori Masu, Keisuke Yonamine, Kazuaki Miyamoto, Fumisato Kozakai, \\ Kazuki Endo, Yutaka Noda and Kei Ito
}

\begin{abstract}
:
Objective Recently, a new digital peroral cholangioscopy (POCS) system, the SpyGlass DS (SpyDS), has been used for POCS-guided lithotripsy for difficult bile duct stones (DBDSs). The aim of this retrospective study was to compare the efficacy of SpyDS-guided electrohydraulic lithotripsy (EHL) for DBDS with that of a conventional digital cholangioscope.

Methods Seventeen consecutive patients who had undergone POCS-guided EHL for DBDS with the SpyDS between October 2015 and January 2019 were enrolled in this study group (SpyDS group) using a prospectively maintained database. Fifteen other consecutive patients who had undergone POCS-guided EHL with a conventional digital cholangioscope (CHF-B260) just prior to the introduction of the SpyDS between December 2006 and September 2015 were analyzed as a control group (CHF group). The main outcome measurement was the total procedure time to complete stone removal.

Results The rate of complete stone removal was $100 \%$ for both groups. The mean total procedure time for the SpyDS group was significantly shorter than that for the CHF group $(67 \pm 30$ minutes vs. $107 \pm 64$ minutes, $\mathrm{p}=0.038$ ). The mean number of endoscopic sessions for the SpyDS group was significantly lower than that for the CHF group $(1.35 \pm 0.49$ vs. $2.00 \pm 0.85, \mathrm{p}=0.037)$. There were no significant differences in the rate of adverse events between the two groups.

Conclusion The SpyDS appears useful for decreasing the procedure time and number of endoscopic sessions for complete stone removal in POCS-guided EHL for DBDS compared with a conventional digital cholangioscope.
\end{abstract}

Key words: endoscopic retrograde cholangiopancreatography, cholangiopancreatoscopy, single-operator, difficult stone

(Intern Med 59: 1925-1930, 2020)

(DOI: 10.2169/internalmedicine.4463-20)

\section{Introduction}

Most bile duct stones (BDSs) can be removed with standard techniques of endoscopic retrograde cholangiopancreatography (ERCP) using a basket catheter, balloon catheter, or mechanical lithotripter following endoscopic sphincterotomy (EST) and/or endoscopic papillary large-balloon dila- tion (EPLBD). Difficult bile duct stones (DBDSs) are defined as BDSs that cannot be removed using standard ERCP techniques (1). DBDSs include huge, multiple, impacted, intrahepatic, and cystic duct stones that cannot be technically captured using standard devices, such as a basket and balloon catheters and mechanical lithotripter. Electrohydraulic lithotripsy (EHL) or laser lithotripsy (LL) with peroral cholangioscopy (POCS) guidance, EHL/LL with percutaneous 


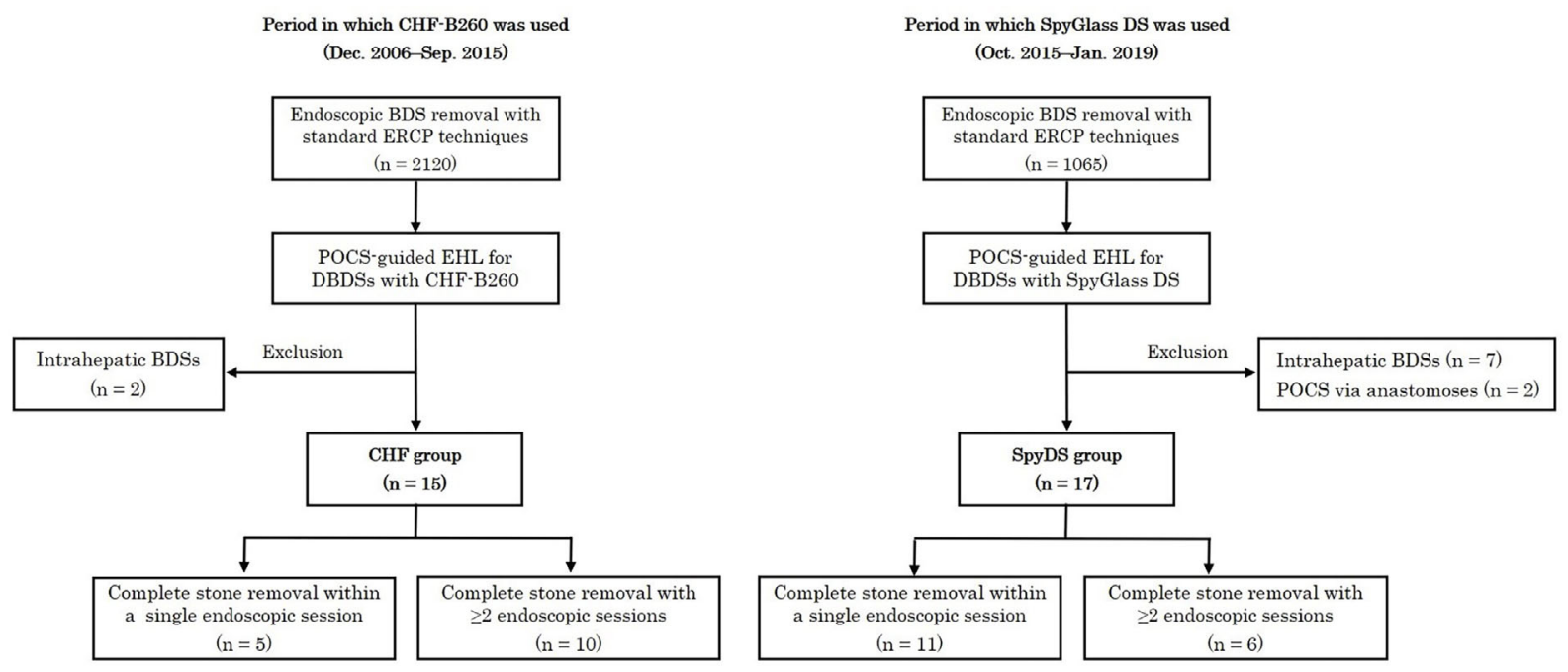

Figure 1. Flowchart of the patients in this study. BDS: bile duct stone, ERCP: endoscopic retrograde cholangiopancreatography, POCS: peroral cholangioscopy, DBDS: difficult bile duct stone, CHF: CHF-B260, SpyDS: SpyGlass DS

transhepatic cholangioscopy guidance, extracorporeal shock wave lithotripsy (ESWL), or surgical stone removal are options for treating such DBDSs $(1,2)$.

Recently, a new digital cholangioscope, the SpyGlass DS Direct Visualization System (SpyDS) (Boston Scientific, Marlborough, USA), has become available. The SpyDS has several benefits for POCS-guided EHL/LL for DBDS: (i) tapered tip for easier insertion into the bile duct, (ii) a digital field of view of $120^{\circ}$, (iii) 2 dedicated irrigation channels, and (iv) a 1.2-mm diameter working channel that enables suction even when a 1.9-Fr lithotripsy probe is mounted through a 3-port adaptor. Before the SpyDS was available, the first-generation SpyGlass system (Boston Scientific) or conventional digital cholangioscopes, such as the CHF-B260 scope (Olympus, Tokyo, Japan) and FCP-9P scope (Pentax Medical, Tokyo, Japan), had been used for POCS-guided EHL/LL (3-5).

Recently, the efficacy of SpyDS-guided EHL/LL for DBDS has been reported (6-9). However, there have been no reports comparing SpyDS with conventional digital cholangioscopes for DBDS. Therefore, we conducted this retrospective comparative study in which the efficacy of SpyDSguided EHL for DBDS was compared with that of a conventional digital cholangioscope.

\section{Materials and Methods}

\section{Patients}

Between December 2006 and September 2015, all POCSguided EHL for DBDS at Sendai City Medical Center were performed using a CHF-B260. However, since the SpyDS system became available in October 2015, the SpyDS has been used for all procedures of POCS-guided EHL. Seventeen consecutive patients who had undergone POCS-guided EHL for DBDS with the SpyDS between October 2015 and
January 2019 were identified using a prospectively maintained database and enrolled in the study group (SpyDS group) (Fig. 1). Fifteen other consecutive patients who had undergone POCS-guided EHL using a CHF-B260 between December 2006 and September 2015 were analyzed as a control group (CHF group) (Fig. 1). A retrospective study was conducted comparing the two groups.

Indications for POCS-guided EHL in this study were DBDSs, which were defined as BDSs that could not be removed with standard ERCP techniques, including mechanical lithotripsy and EPLBD. DBDSs included stones deemed too large, stones at the cystic duct confluence, and Mirizzi syndrome with biliobiliary fistula. All cases of intrahepatic BDSs were excluded from this study, even if they could be removed using POCS-guided EHL (Fig. 1), because intrahepatic BDSs were extremely heterogeneous, making it difficult to compare the outcomes of endoscopic treatment. For example, some cases needed hepatectomy for a malignant intrahepatic biliary stricture without endoscopically complete stone removal, and other cases had multiple and severe biliary strictures due to sclerosing cholangitis, making complete stone removal extremely difficult. In addition, patients who had undergone POCS-guided EHL through the anastomoses, such as hepaticogastrostomy or choledochoduodenostomy, were also excluded (Fig. 1).

The presence of BDSs was confirmed using imaging examinations, including abdominal ultrasonography (US), computed tomography (CT), magnetic resonance imaging (MRI), and/or endoscopic ultrasound (EUS), before endoscopic stone removal. This study was approved by the Institutional Review Board of Sendai City Medical Center (approval number: 2016-0032).

\section{Procedures}

All patients received intravenous antibiotics 30 minutes before and 6 hours after ERCP. ERCP was performed using 

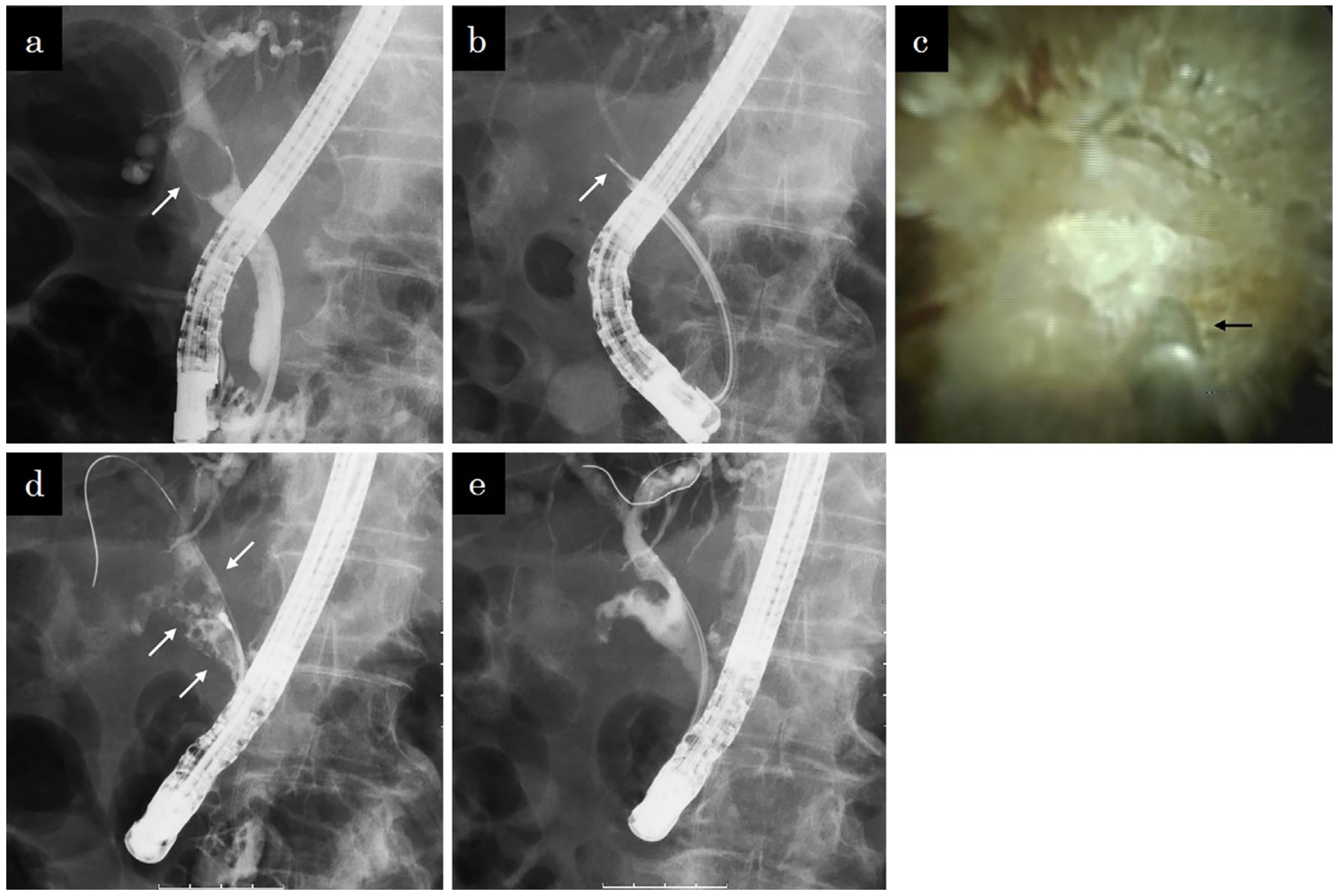

Figure 2. A case of a stone at the cystic duct confluence. a: Cholangiographic image showing a large stone (arrow) impacted in the cystic duct confluence. b: Fluoroscopic image showing an EHL probe (arrow) through the SpyGlass DS in the common bile duct. c: Cholangioscopic image showing a probe (arrow) located in front of the stone at the cystic duct confluence. $d$ : Cholangiographic image showing fragmented stones (arrows) in the bile and cystic ducts. e: Cholangiographic image after achieving complete stone removal. There were no filling defects in either the bile or cystic ducts.

a duodenoscope (TJF-260V; Olympus) under moderate sedation with intravenous administration of midazolam and pentazocine. Standard stone removal techniques, including the use of mechanical lithotripsy following EST and/or EPLBD, were attempted in the same or previous session before applying POCS-guided EHL in all patients. EPLBD was applied in cases with dilated $(\geq 12 \mathrm{~mm})$ distal bile ducts at the endoscopist's discretion.

The procedures for both types of cholangioscope were performed by two endoscopists via mother-baby methods, with one endoscopist operating a duodenoscope and the other a cholangioscope. Experts who had conducted more than 500 ERCP procedures performed all endoscopic procedures as an operator of either the duodenoscope or the cholangioscope. After inserting the cholangioscope into the bile duct with or without guidewire assistance, BDSs were directly visualized using the cholangioscope with saline irrigation. For the SpyDS group, saline was infused into the bile duct through the dedicated irrigation channels using a foot switch-controlled water pump. For the CHF group, saline was manually infused through the working channel using a syringe with a three-port adaptor attached because the CHFB260 scope had only one channel for both irrigation and suction. An AUTOLITH system (Northgate Technologies, Elgin, USA) with an electrohydraulic shock-wave generator and 1.9-Fr catheter probe was used for EHL. After stone fragmentation using EHL under cholangioscopic guidance, fragmented stones were removed using standard devices, such as a basket or balloon catheter. Complete stone removal was confirmed based on cholangioscopic and/or cholangiographic findings. If stones were not completely removed during the first session, a plastic stent was temporarily placed to avoid biliary obstruction, and endoscopic procedures were repeated with intervals of two to seven days until complete removal was achieved. All patients were hospitalized for at least 24 hours after endoscopic treatment to evaluate adverse events. The applied standard ERCP techniques and equipment including EHL and fluoroscopic system other than POCS type were the same for both groups throughout.

Fig. 2 shows a case with a large stone at the cystic duct confluence that was successfully treated using SpyDSguided EHL.

\section{Outcomes and definitions}

The main outcome measurement was the total procedure time to complete stone removal for each group. In addition, the rate of complete stone removal, total procedure time taken in POCS, number of endoscopic sessions, and adverse events of the two groups were compared. Total procedure time was defined as the total time required over all endoscopic sessions to achieve complete stone removal, meas- 
Table 1. Patient Characteristics.

\begin{tabular}{lccc}
\hline & $\begin{array}{c}\text { CHF group } \\
(\mathrm{n}=15)\end{array}$ & $\begin{array}{c}\text { SpyDS group } \\
(\mathrm{n}=17)\end{array}$ & p value \\
\hline Age (years), mean \pm SD & $75.7 \pm 10.5$ & $67.1 \pm 12.9$ & 0.049 \\
Sex, male/female & $4 / 11$ & $9 / 8$ & 0.131 \\
Reasons for the failure to remove stones using standard techniques & & & 0.445 \\
$\quad$ Large stones & $10(66.7 \%)$ & $10(58.8 \%)$ & \\
$\quad$ Stones at cystic duct confluence & $4(26.7 \%)$ & $6(35.3 \%)$ & \\
$\quad$ Mirizzi syndrome with biliobiliary fistula & $1(6.7 \%)$ & $1(5.9 \%)$ & \\
Largest stone size (mm), mean \pm SD & $20.5 \pm 6.8$ & $19.8 \pm 6.6$ & 0.788 \\
Number of stones, mean \pm SD & $1.8 \pm 1.2$ & $2.5 \pm 2.1$ & 0.551 \\
Diameter of common bile duct (mm), mean $\pm S D$ & $16.2 \pm 5.4$ & $15.1 \pm 4.9$ & 0.533 \\
Ampullary interventions prior to stone removal & & & 0.208 \\
$\quad$ EST & $14(93.3 \%)$ & $13(76.5 \%)$ & \\
EPLBD with EST & $1(6.7 \%)$ & $4(23.5 \%)$ & \\
\hline
\end{tabular}

EST: endoscopic sphincterotomy, EPLBD: endoscopic papillary large balloon dilation

ured from the first insertion of the cholangioscope in the first session to the removal of the duodenoscope in the session at which complete stone removal was achieved. For patients who had to undergo multiple endoscopic sessions to complete stone removal, the times of each session were added to determine the total procedure time. For patients who needed biopsies of indeterminate biliary strictures with cholangioscopic or fluoroscopic guidance, the time taken for biopsy procedures was excluded from the total procedure time. The procedure time was determined by analyzing the video recorded during all endoscopic procedures. Procedurerelated adverse events and their severity were defined following the Consensus Criteria (10). The number of stones was confirmed using cholangiography, and the diameter of the stones and common bile duct were measured in reference to the diameter of the endoscope. EPLBD was defined as mechanical dilation of the major duodenal papilla using a balloon with a diameter $\geq 12 \mathrm{~mm}$.

\section{Statistical analyses}

Continuous variables are represented as mean values with standard deviations, whereas categorical variables are reported as patient numbers and percentages. Continuous variables were compared using unpaired Student's $t$ - or MannWhitney tests, and categorical variables were compared using the chi-square or Fisher's exact tests, as appropriate.

A $p$ value of $<0.05$ was considered to be statistically significant. The SPSS software program (version 24; IBM Japan, Tokyo, Japan) was used for all analyses.

\section{Results}

A flowchart of the patients in this study is shown in Fig. 1. The patient characteristics of the two groups are shown in Table 1. There were no significant differences in reasons for the failure to remove stones using standard techniques, size and number of stones, diameter of common bile duct, and ampullary interventions prior to stone removal be- tween the two groups.

The outcomes of the two groups are shown in Table 2. The rate of complete stone removal was $100 \%$ for both groups. The mean total procedure time for the SpyDS group was significantly shorter than that for the CHF group (67 \pm 30 minutes vs. $107 \pm 64$ minutes, $\mathrm{p}=0.038$ ). The mean total procedure time for POCS for the SpyDS group was significantly shorter than that for the CHF group ( $28 \pm 13$ minutes vs. $50 \pm 36$ minutes, $\mathrm{p}=0.044$ ). The mean number of endoscopic sessions for the SpyDS group was significantly lower than that for the CHF group $(1.35 \pm 0.49$ vs. $2.00 \pm 0.85, \mathrm{p}=$ $0.037)$. There were no significant differences in the rate of adverse events between the two groups. With respect to the severity of the adverse events, two cases of cholangitis in the SpyDS group and two of three cases of cholangitis in the $\mathrm{CHF}$ group were of moderate grade, whereas the others were of mild grade. All patients developing adverse events recovered with conservative treatment with medications.

\section{Discussion}

To our knowledge, this is the first study to compare the outcomes of SpyDS-guided EHL/LL for DBDSs with those of a conventional digital cholangioscope. Our study showed that using the SpyDS, compared with a conventional digital cholangioscope, significantly decreased the procedure time and number of endoscopic sessions for complete stone removal in POCS-guided EHL for DBDS, although this was a single-center retrospective study with a relatively small population.

There are several functional differences between the SpyDS and CHF-B260 contributing to the superiority of the SpyDS in POCS-guided EHL for DBDSs. The SpyDS scope has greater manipulability and a wider visual field with 4way tip deflection and $120^{\circ}$ field of view, compared with the CHF-B260 scope and its 2-way tip deflection and $90^{\circ}$ field of view. These differences make it easier to clearly visualize stones, which is necessary when performing EHL, 
Table 2. Outcomes.

\begin{tabular}{lccc}
\hline & $\begin{array}{c}\text { CHF group } \\
(\mathrm{n}=15)\end{array}$ & $\begin{array}{c}\text { SpyDS group } \\
(\mathrm{n}=17)\end{array}$ & $\mathrm{p}$ value \\
\hline Complete stone removal & $15(100 \%)$ & $17(100 \%)$ & N. A. \\
Total procedure time to complete stone removal (min), mean \pm SD & $107 \pm 64$ & $67 \pm 30$ & 0.038 \\
Total procedure time taken for POCS (min), mean \pm SD & $50 \pm 36$ & $28 \pm 13$ & 0.044 \\
Number of endoscopic sessions to complete stone removal & & & \\
mean \pm SD & $2.00 \pm 0.85$ & $1.35 \pm 0.49$ & 0.037 \\
1 & $5(33.3 \%)$ & $11(64.8 \%)$ & \\
2 & $5(33.3 \%)$ & $6(35.2 \%)$ & \\
3 & $5(33.3 \%)$ & $0(0 \%)$ & \\
Adverse events & & & \\
Overall & $3(20.0 \%)$ & $4(23.5 \%)$ & 0.576 \\
Cholangitis & $3(20.0 \%)$ & $2(11.7 \%)$ & 0.650 \\
Pancreatitis & $0(0 \%)$ & $1(5.9 \%)$ & 0.531 \\
Cholecystitis & $0(0 \%)$ & $1(5.9 \%)$ & 0.531 \\
\hline POCS: per
\end{tabular}

POCS: peroral cholangioscopy, N.A.: not applicable

with a SpyDS than with the CHF-B260. Furthermore, the SpyDS scope has two dedicated irrigation channels separated from the working channel, which enables unimpeded saline irrigation during lithotripsy, even when an EHL probe is mounted through the working channel. In contrast, the CHF-B260 scope has only one channel for both irrigation and device insertion, which drastically limits saline irrigation when an EHL probe is mounted through the channel. These functional advantages of the SpyDS over the CHF-B 260 likely helped reduce the procedure time for POCSguided EHL for DBDSs.

However, the CHF-B260 still has several advantages over the SpyDS that can help improve the outcomes of POCSguided EHL/LL. The image quality of the CHF-B260 is much higher than that of the SpyDS $(11,12)$. Kanno et al. (12) reported that the ability to make an optical diagnosis of the lateral extent of extrahepatic cholangiocarcinoma through POCS observation was higher with the CHF-B260 than with the SpyDS due to the differences in the image quality. However, excellent manipulability, a wide visual field, and sufficient irrigation capability of POCS appear to be more important for improving the outcomes of POCSguided EHL/LL than a high image quality.

Mizrahi et al. (13) retrospectively compared the outcomes of POCS-guided EHL for DBDS using the SpyDS and a first-generation SpyGlass system. They reported that the mean procedure time and radiation doses for the SpyDS group were significantly lower than those for the firstgeneration SpyGlass group $(49 \pm 17$ minutes vs. $57 \pm 21$ minutes and $361 \pm 250 \mathrm{mGy}$ vs. $620 \pm 452 \mathrm{mGy}$, respectively) and that the rate of complete stone clearance in the first session for the SpyDS group was significantly higher than for the first-generation SpyGlass group (83\% vs. 58\%). However, this seems to be a matter of course, since the SpyDS was developed from the first-generation SpyGlass by the same corporation (Boston Scientific) with functional improvements over all aspects, including the image quality, manipu- lability, and system setup (11, 14-16). The CHF-B260, by contrast, is completely different from the SpyDS. Therefore, our study comparing these two digital cholangioscopes is more valuable, and our results suggest that the SpyDS is currently the optimal cholangioscope for POCS-guided EHL for DBDS.

In May 2019, a new-generation digital POCS with the model number 'CHF-B290' developed based on the CHF-B 260 by Olympus became commercially available in Japan, with an October 2019 release in Europe. The CHF-B290 has notable improvements over the CHF-B260, including the diameter of the working channel and scope durability. The CHF-B290 has a 1.3-mm diameter working channel (vs, 1.2 $\mathrm{mm}$ for CHF-B260), which improves the ability to irrigate and perform suction through the working channel, especially when an EHL probe is mounted (17). Furthermore, the CHF-B260 was extremely fragile and often malfunctioned, requiring repair before reuse (5); the durability of the CHFB290 has been markedly improved. Future clinical reports on the use of the CHF-B290 to treat biliary disease, including DBDS, are warranted.

The rate of overall adverse events for the SpyDS group in this study was $24 \%$, which is higher than the rates described in previous reports, which ranged from $1.5 \%$ to $14 \%(6-9,18)$. This may be due to the fact that our study population included more difficult-to-manage cases than the previous studies did. For example, Kamiyama et al. (8) reported a median procedure time, defined as in our study, of 27 minutes, which is markedly shorter than ours (67 minutes), and a rate of adverse events of $14 \%$. Cholangitis is the most common adverse event of POCS-guided EHL/LL for DBDSs due to increased biliary pressure caused by frequent saline irrigation into the bile duct during the procedures $(7,18,19)$, and this is consistent with our study, which saw five cases of cholangitis out of seven cases with adverse events. Although there have been concerns about frequent cholangitis in the SpyDS group because irrigation 
is easier, the adverse event rates, including cholangitis, for the groups in our study were similar.

Several limitations associated with the present study warrant mention. First, it was a single-center retrospective study with a small population. It seemed inappropriate to prospectively carry out a randomized control study to compare a SpyDS and a conventional digital cholangioscope for POCSguided lithotripsy because the superiority of the SpyDS was apparent at the time it became available. Second, the study periods for the two groups were completely different, which may have led to selection bias, learning curve bias, and bias in the differences in the devices other than POCS. Although the criteria of indication for POCS-guided EHL were the exact same for both groups, the actual indications for POCSguided EHL were left to the endoscopist's discretion. The learning curves of the endoscopists may have also influenced the results of this study, since the procedures using the SpyDS were performed after those using the CHF-B260. Furthermore, devices for stone removal other than POCS or EHL systems, such as basket and balloon catheters and a mechanical lithotripter, may have differed somewhat between the groups due to developmental advances in these devices, which may have influenced the results of some of the cases. Finally, the cost effectiveness was not evaluated in this study. Using the CHF-B260 may be more cost-effective, since SpyDS scopes are disposable while the CHF-B260 ones are reusable.

In conclusion, the SpyDS appears useful for decreasing the procedure time and number of endoscopic sessions for complete stone removal in POCS-guided EHL for DBDS compared with a conventional digital cholangioscope. The further development of devices, including cholangioscopes, in the future will help improve the outcomes of POCSguided EHL/LL for DBDS.

The authors state that they have no Conflict of Interest (COI).

\section{References}

1. Yasuda I, Itoi T. Recent advances in endoscopic management of difficult bile duct stones. Dig Endosc 25: 376-385, 2013.

2. Manes G, Paspatis G, Aabakken L, et al. Endoscopic management of common bile duct stones: European Society of Gastrointestinal Endoscopy (ESGE) guideline. Endoscopy 51: 472-491, 2019.

3. Itoi T, Sofuni A, Itokawa F, Shinohara Y, Moriyasu F, Tsuchida A. Evaluation of residual bile duct stones by peroral cholangioscopy in comparison with balloon-cholangiography. Dig Endosc 22: S85S89, 2010.

4. Maydeo A, Kwek BE, Bhandari S, Bapat M, Dhir V. Singleoperator cholangioscopy-guided laser lithotripsy in patients with difficult biliary and pancreatic ductal stones (with videos). Gastrointest Endosc 74: 1308-1314, 2011.
5. Ishida Y, Itoi T, Okabe Y, et al. Types of peroral cholangioscopy: how to choose the most suitable type of cholangioscopy. Curr Treat Options Gastroenterol 14: 210-219, 2016.

6. Wong JC, Tang RS, Teoh AY, Sung JJ, Lau JY. Efficacy and safety of novel digital single-operator peroral cholangioscopyguided laser lithotripsy for complicated biliary stones. Endosc Int Open 05: E54-E58, 2017.

7. Brewer Gutierrez OI, Bekkali NLH, Raijman I, et al. Efficacy and safety of digital single-operator cholangioscopy for difficult biliary stones. Clin Gastroenterol Hepatol 16: 918-926, 2018.

8. Kamiyama R, Ogura T, Okuda A, et al. Electrohydraulic lithotripsy for difficult bile duct stones under endoscopic retrograde cholangiopancreatography and peroral transluminal cholangioscopy guidance. Gut Liver 12: 457-462, 2018.

9. Canena J, Lopes L, Fernandes J, et al. Outcomes of singleoperator cholangioscopy-guided lithotripsy in patients with difficult biliary and pancreatic stones. GE Port J Gastroenterol 26: 105-113, 2019.

10. Cotton PB, Eisen GM, Aabakken L, et al. A lexicon for endoscopic adverse events: report of an ASGE workshop. Gastrointest Endosc 71: 446-454, 2010.

11. Tanaka R, Itoi $T$, Honjo $M$, et al. New digital cholangiopancreatoscopy for diagnosis and therapy of pancreaticobiliary diseases (with videos). J Hepatobiliary Pancreat Sci 23: 220-226, 2016.

12. Kanno Y, Koshita S, Ogawa $T$, et al. Peroral cholangioscopy by SpyGlass DS versus CHF-B260 for evaluation of the lateral spread of extrahepatic cholangiocarcinoma. Endosc Int Open 6: E1349E1354, 2018.

13. Mizrahi M, Khoury T, Wang Y, et al. "Apple Far from the Tree": comparative effectiveness of fiberoptic single-operator cholangiopancreatoscopy (FSOCP) and digital SOCP (DSOCP). HPB 20: 285-288, 2018.

14. Shah R, Neuhaus H, Reddy ND, Parsi MA, Pleskow DK. A Randomized assessment of a semi-disposable, fiberoptic singleoperator cholangioscope with a fully-disposable, digital singleoperator cholangioscope in a bench model. Gastrointest Endosc 83: AB601, 2016.

15. Navaneethan U, Hasan MK, Kommaraju K, et al. Digital, singleoperator cholangiopancreatoscopy in the diagnosis and management of pancreatobiliary disorders: a multicenter clinical experience (with video). Gastrointest Endosc 84: 649-655, 2016.

16. Ogawa T, Ito K, Koshita S, et al. Usefulness of cholangioscopicguided mapping biopsy using SpyGlass DS for preoperative evaluation of extrahepatic cholangiocarcinoma: a pilot study. Endosc Int Open 6: E199-E204, 2018.

17. Ishida $Y$, Itoi $T$, Okabe $Y$. Current status and future perspective in cholangiopancreatoscopy. Curr Treat Options Gastroenterol 17: 327-341, 2019.

18. Jin Z, Wei Y, Tang X, et al. Single-operator peroral cholangioscope in treating difficult biliary stones: a systematic review and meta-analysis. Dig Endosc 31: 256-269, 2019.

19. Sethi A, Chen YK, Austin GL, et al. ERCP with cholangiopancreatoscopy may be associated with higher rates of complications than ERCP alone: a single-center experience. Gastrointest Endosc 73: 251-256, 2011.

The Internal Medicine is an Open Access journal distributed under the Creative Commons Attribution-NonCommercial-NoDerivatives 4.0 International License. To view the details of this license, please visit (https://creativecommons.org/licenses/ by-nc-nd/4.0/).

(C) 2020 The Japanese Society of Internal Medicine Intern Med 59: 1925-1930, 2020 